\title{
The impact of frequency aliasing on spectral method of measuring $T$ wave alternans*
}

\author{
Di-Hu Chen ${ }^{1}$, Sheng Yang ${ }^{1}$ \\ ${ }^{1}$ Department of Precision Machinery \& Instrumentation, University of Science and Technology of China, Hefei 230027, China. \\ Email: yangs@ustc.edu.cn. \\ Received Jan. $5^{\text {th }}, 2009$; revised Feb. $19^{\text {th }}, 2009$; accepted Feb. $20^{\text {th }}, 2009$.
}

\begin{abstract}
In this paper we investigate frequency aliasing in spectral method of measuring $T$ wave alternans, which may lead a high false positive rate. Microvolt $T$ wave alternans(TWA) has been evaluated as a means of predicting occurrence of ventricular tachyarrhythmia events and its association with the genesis of ventricular arrhythmias has been demonstrated. Nowadays, spectral method is one of the most widely used procedures for measurement of microvolt TWA. In our study, based on the sampling theory, the alternans frequency 0.5 cycles/beat, at which the power of the spectrum is used to calculated the $\mathrm{V}_{\text {alt }}$ and $\mathrm{K}$ score (these two parameters indicate the TWA), is equal to the nyquist frequency. Thus this generates frequency aliasing which will make the power at the alternans frequency $\left(P_{0.5}\right)$ be two times of the real magnitude of the original spectrum amplitude. With the assumption that the noise spectrum follows the normal distribution, in spectral method of measuring $T$ wave alternans, the measuring standard $K$ score $>3$ to consider the $T$ wave alternans significant is only with a $p<0.133$. By change the standard to $K$ score $>6$ can solve this problem and make the $p$ value to $p<0.0027$.
\end{abstract}

Keywords: TWA, Sampling, Spectral method, Frequency Aliasing

\section{INTRODUCTION}

Sudden cardiac death (SCD) is the leading cause of cardiovascular mortality in the developed countries [1]. There is no an effective diagnostic method to identify patients at high risk for SCD. Though many non-invasive tests related to high-risk of SCD such as frequent and complex ventricular arrhythmias in 24-hour Holter monitoring, ventricular late potentials in signal-average ECG, low heart rate variability, and increased dispersion of repolarization are introduced, the positive predictive

\footnotetext{
${ }^{*}$ This work was support by the National Natural Science Foundation
} of China (60571034) value of these tests is too low to consider them as sufficient to make a decision about specific treatment, especial defibrillator implantation. Recently risk stratification research has been focused on microvolt $\mathrm{T}$ wave alternans, which is considered as a promising clinical marker of arrhythmic events [2].

Microvolt T wave alternans (TWA), also called repolarization alternans, is a phenomenon appearing in the electrocardiogram (ECG) as a consistent fluctuation in the repolarization morphology on an every-other-beat basis [3]. Microvolt TWA has been evaluated prospectively in a variety of patient populations as a means of predicting occurrence of ventricular tachyarrhythmia events and its association with the genesis of ventricular arrhythmias has been demonstrated [4].

In measuring of TWA, spectral method is a widely used method. This method uses a certain measurements taken on corresponding points of some consecutive $T$ wave to compute a spectrum. And then two parameters: the alternans voltage $\left(V_{\text {alt }}\right)$ and alternans ratio ( $K$ score) are calculated from this spectrum. These two parameters indicate whether the TWA is significant.

This paper investigates frequency aliasing (also called aliasing in short) in spectral method of measuring $\mathrm{T}$ wave alternans, which may lead a high false positive rate. In section II we take a brief view of the spectral method of measuring $\mathrm{T}$ wave alternans. In section III, we introduce the sampling theory and frequency aliasing and in section IV, we investigate frequency aliasing in the spectral method.

\section{SPECTRAL METHOD OF MEASURING T WAVE ALTERNANS}

Until now, two main techniques have been applied for measurement of microvolt TWA in clinical setting: fast Fourier Transform (FFT) spectral method and modified moving average (MMA) analysis method [4]. The FFT spectral method which was developed at Massachusetts Institute of Technology by Dr. Richard J. Cohen [5, 6, 7] is the most widely used procedure. This technique uses 128 measurements taken on corresponding points of 128 consecutive $T$ waves to compute a spectrum. Each $T$ wave is measured at the same time relative to the QRS complex [8]. For the spectrum is created by measure- 
ments taken once per beat, its frequencies are in the units of cycles/beat. The point on the spectrum corresponding to exactly 0.5 cycles/beat indicates the level of alternation of $\mathrm{T}$ wave waveform [8].

Two measurements are obtained form the analysis: the alternans voltage $\left(V_{\text {alt }}\right)$ and alternans ratio ( $K$ score). The $V_{\text {alt }}$ measured in $u \mathrm{~V}$, represents the square root of alternans power which is defined as the difference between the power at the alternans frequency $(0.5$ cycles/beat) and the power at the noise frequency $(0.44$ and 0.49 cycles/beat). And also, it corresponds to the root mean square difference in the voltage between the overall mean beat and either the odd-numbered or even- numbered beats. The alternans ratio $K$ score is calculated as the ratio of the alternans power divided by the standard deviation of the noise in the reference frequency band. See bellow

$$
\begin{gathered}
V_{\text {alt }}=P_{0.5}-\mu \\
K \text { score }=\frac{P_{0.5}-\mu}{\sigma}
\end{gathered}
$$

where $\mathrm{P}_{0.5}$ is the power at 0.5 cycles/beat, $\mu$ and $\sigma$ are the mean and standard deviation. When the alternans power is $>3 \mathrm{SD}$ above the noise level ( $K$ score $>3$ ), alternans is considered significant in statistic.

\section{NYQUIST FREQUENCY AND FRE- QUENCY ALIASING IN MEASURING T WAVE ALTERNANS}

Consider a continuous-time signal $f(t)$. We define sampling as the generation of an ordered number sequence by taking values of $f(t)$ at specified instants of time [9]. In most cases continuous-time signals are sampled at equal increments of time. The sample increment, called the sample period, is usually denoted as $T_{s}$. Therefore, the sampled signal values available in the computer are $f\left(n T_{s}\right)$, where $n$ is an integer.

Figure 1 shows the ideal impulse sampling operation. This is seen to be a modulation process, in which the carrier signal $\delta_{T}(t)$ is defined as the train of impulse function:

$$
\delta_{T}(t)=\sum_{n=-\infty}^{\infty} \delta\left(t-n T_{S}\right)
$$

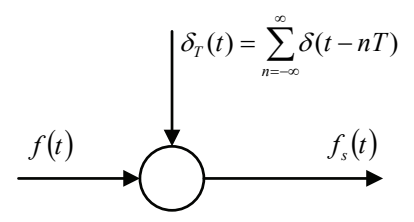

Figure 1. Impulse sampling.

The output of the modulator, denoted by $f_{s}(t)$ is given by

$$
f_{s}(t)=f(t) \delta_{T}(t)=\sum_{n=-\infty}^{\infty} f\left(n T_{s}\right) \delta\left(t-n T_{s}\right)
$$

We begin to investigate the characteristics of the sampling operation in Figure 1 by taking the Fourier transform of $f_{s}(t)$. For Fourier transform, we can easy get

$$
\delta_{T}(t)=\sum_{n=-\infty}^{\infty} \delta\left(t-n T_{s}\right) \stackrel{F}{\longleftrightarrow} \omega_{s} \sum_{k=-\infty}^{\infty} \delta\left(\omega-k \omega_{s}\right)
$$

where $\omega_{s}=\frac{2 \pi}{T_{s}}$ is the sampling frequency in radians/second. The sampling frequency in hertz is giver by $f_{s}=\frac{1}{T_{S}}$; therefore, $\omega_{s}=2 \pi f_{s}$. For multiplication in the time domain results in convolution in the frequency domain. Then from (2) and (3),

$$
\begin{array}{r}
F_{s}(\omega)=\frac{1}{2 \pi} F(\omega) *\left[\omega_{s} \sum_{k=-\infty}^{\infty} \delta\left(\omega-k \omega_{s}\right)\right] \\
=\frac{1}{T_{s}} \sum_{k=-\infty}^{\infty} F(\omega) * \delta\left(\omega-k \omega_{s}\right)
\end{array}
$$

where $F(\omega)$ is the Fourier transform of $f(t)$ and $F_{s}(\omega)$ is the Fourier transform of $f_{s}(t)$. Because of the convolution property of the impulse function,

$$
F(\omega) * \delta\left(\omega-k \omega_{s}\right)=F\left(\omega-k \omega_{s}\right)
$$

Thus the Fourier transform of the impulse-modulated signal (2) is given by

$$
F_{s}(\omega)=\frac{1}{T_{s}} \sum_{k=-\infty}^{\infty} F\left(\omega-k \omega_{s}\right)
$$

Frequency domain characteristics of the sampling operation can be derived from this result.

From (6) we see that the effect of sampling $f(t)$ is to replicate the frequency spectrum of $F(\omega)$ about the frequencies $k \omega_{s}, k= \pm 1, \pm 2, \pm 3 \ldots$ This result is show in Figure 2(b) for the signal of Figure 2(a).

The frequency $\omega_{s} / 2$ is called the Nyquist frequency and the Shannon sampling frequency. One of the requirements for sampling is that the sampling frequency
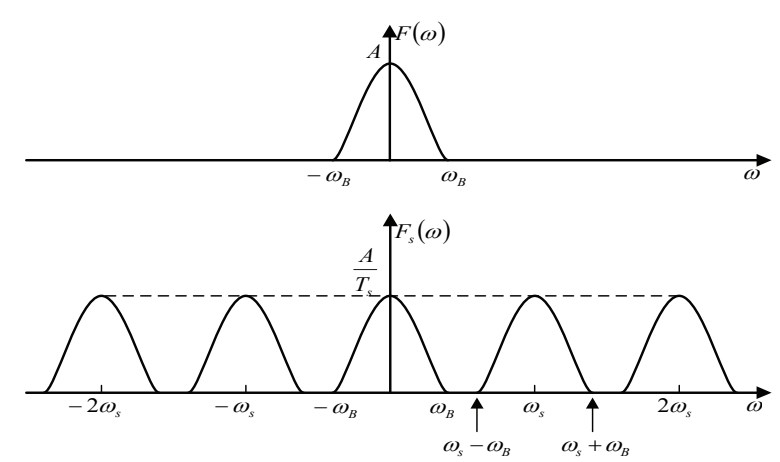

Figure 2. The frequency spectrum of a sampled signal. 
must be chosen such that $\omega_{s}>2 \omega_{M}$, where $\omega_{M}$ is the highest frequency in the frequency spectrum of the signal to be sampled.

From part 2 we know, in spectral analysis of microvolt $\mathrm{T}$ wave alternans, the sampling frequency is $1 \mathrm{cy}-$ cles/beat and the alternans frequency is 0.5 cycles/beat, which is exactly 0.5 of the sampling frequency. This is also the Nyquist frequency. In sampling theory, input-signal frequencies that exceed the Nyquist frequency are aliased. That is, they are folded back or replicated at other positions in the spectrum above and below the Nyquist frequency. (See Figure 3)

So in spectral method of measuring $\mathrm{T}$ wave alternans, power at the alternans frequency $\left(\mathrm{P}_{0.5}\right)$ which is used to indicate the level of alternation of $\mathrm{T}$ wave waveform is two times of the real magnitude of the original spectrum at 0.5 cycles/beat.

\section{DISCUSSION}

In spectral method of measuring $\mathrm{T}$ wave alternans, as mentioned in part 2, the $\mathrm{T}$ wave alternans is considered significant when the $\mathrm{K}$ score is higher than 3 , while the $\mathrm{K}$ score represents the ratio of the alternans power and the standard noise power deviation. In order to explain why this rule works, let's take a look at the normal distribution.

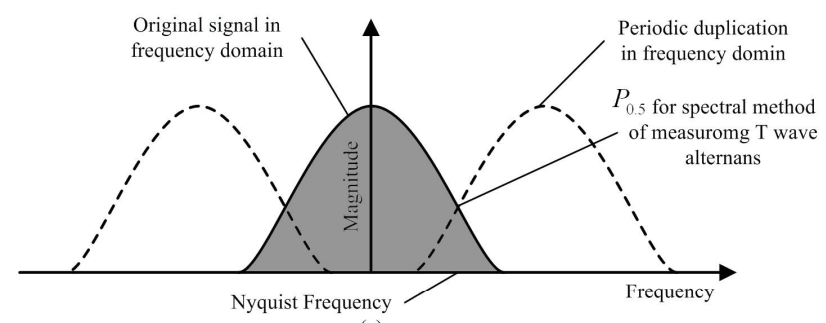

(a)

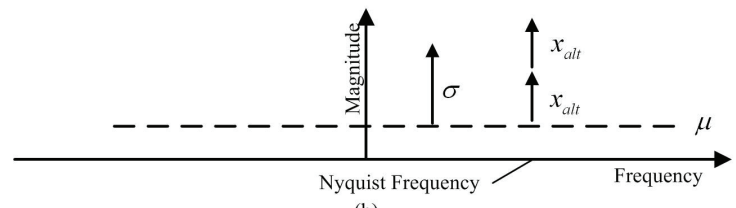

(b)

Figure 3. (a) Input signal frequencies exceed the Nyquist frequency are aliased.(b) With the frequency aliasing, P0.5 is two times of the real magnitude.

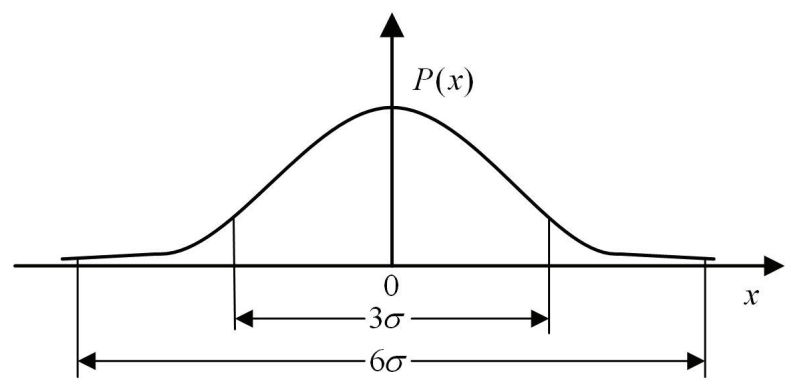

Figure 4. Probability density function of the normal distribution.
A normal distribution in a variate $\mathrm{X}$ with mean $\mu$ and variance $\sigma^{2}$ is a statistic distribution with probability function

$$
P(x)=\frac{1}{\sigma \sqrt{2 \pi}} e^{-(x-\mu)^{2} /\left(2 \sigma^{2}\right)}
$$

on the domain $x \in(-\infty, \infty)$. The importance of the normal distribution as a model of quantitative phenomena in the natural and behavioral sciences is due in part to the central limit theorem. Many measurements, ranging from psychological to physical phenomena can be approximated, to varying degrees, by the normal distribution. In the spectral method of measuring $\mathrm{T}$ wave alternans, the noise of the power spectrum can be assumed to be normal. In normal distribution, if $(x-u) / \sigma>3$, then the $\mathrm{x}$ is statistically significant with the probability $(p<0.0027)$ due to chance. This can be easily calculated via (9) and this probability is also called the false positive rate. When there is frequency aliasing, let $x_{\text {alt }}$ be the real alternans power, and assume that the distribution of the noise spectrum is normal no matter whether there is $\mathrm{T}$ wave alternans, then $P_{0.5}=2 x_{\text {alt }}+\mu$ and (2) can be written as

$$
K_{\text {score }}=\frac{2 x_{\text {alt }}+\mu-\mu}{\sigma}=\frac{2 x_{\text {alt }}}{\sigma}>3
$$

This is also $x_{\text {alt }} / \sigma>1.5$, from the table of the standard normal distribution we can get that in this condition the $p$ value is only less than $0.133(p<0.133)$, which means a high false positive rate.

In order to solve this problem, we can change the standard from $K_{\text {score }}>3$ to $K_{\text {score }}>6$. From (10) we can get $p<0.0027$ if consider $\mathrm{T}$ wave alteransn significant when $K_{\text {score }}>6$.

\section{CONCLUSION}

In this paper study, based on the sampling theory, in spectral method of measuring $\mathrm{T}$ wave alternans, the alternans frequency is equal to the nyquist frequency, and this makes frequency aliasing in the power spectrum, which will lead the increase of the false positive rate, from $p<0.0027$ to $p<0.133$. By changing the standard from $K_{\text {score }}>3$ to $K_{\text {score }}>6$ can effectively solve this problem.

\section{REFERENCES}

[1] A. Bay\& and J. Guindo, (1989) Sudden Cardiac Death. Spain: MCR.

[2] J. P. Martinez, S. Olmos and P. Laguna, (2000) Simulation Study and Performance Evaluation ofT-Wave Alternans Detec- 
tor. Proceedings of the 22nd Annual EMBS International Conference, July 23-28, Chicago IL.

[3] J. P. Martínez and S. Olmos, (2005) Methodological Principles of T Wave Alternans Analysis: A Unified Framework. IEEE Transactions On Biomedical Engineering, vol. 52, NO. 4.

[4] B. D. Nearing, R. L. Verrier. (2002) Modified moving average method for $\mathrm{T}-$ wave alternans analysis with high accuracy to predict ventricular fibrillation. J Appl Physiol, 92, 541-49.

[5] D. R. Adam, J. M. Smith, S. Akselrod, S. Nyberg, A. O. Powell, R. J. Cohen. (1984) Fluctuations in T-wave morphology and susceptibility to ventricular fibrillation. J Electrocardiol, 17, 209-18.
[6] A. L. Ritzenberg, D. R. Adam, R. J. Cohen. (1984) Period multiplying-evidence for nonlinear behavior of the canine heart. Nature, 307, 159-61.

[7] J. M. Smith, E. A. Clancy, C. R. Valeri, J. N. Ruskin, R. J. Cohen. (1988) Electricalalternans and cardiac electrical instability. Circulation, 77, 110-21.

[8] D. M. Bloomfield, S. H. Hohnloser, R. J. Cohen. (2002) Interpretation and classification of microvolt $\mathrm{T}^{-}$-wave alternans tests. J Cardiovasc Electrophysiol, 13:502- 12.

[9] C. L. Phillips, J. M. Parr and E. A. Riskin. (2004) Signal, System and Transform. China Machine Press, Beijing. 